\title{
Black or Brown Pigment and Cholesterol Gallstones Formation Among Patients that Underwent Gastrectomy for Cancer
}

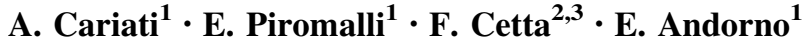

Published online: 2 March 2017

(C) Société Internationale de Chirurgie 2017

\section{Dear Editor,}

We read the interesting article of Kimura et al. [1] on the risk of onset of symptomatic gallstones disease among patients that underwent gastrectomy for cancer. Authors reported the onset of nine pigment gallstones over ten patients with cholecystolithiasis (90\%); (cholesterol gallstones 10\%) and in four patients over four affected by common bile duct gallstones (100\%) [1]. In the discussion chapter, the possible mechanisms of gallstones formation have been described. However, Kimura et al. [1] did not attempt at distinguishing black pigment gallstones from brown pigment gallstones. Previous studies [2] demonstrated that black pigment gallstones account for $3 / 7$ (42.8\%) of the cases, cholesterol gallstones for $2 / 7(28.5 \%)$ and brown pigment gallstones for $2 / 7$ $(28.5 \%)$ of the patients. Risk factors for black pigment gallstones formation $[3,4]$ differ from those of brown pigment gallstones formation [5].The main risk factor for brown pigment gallstones formation, usually in the common bile duct, has been bacteriobilia [5] or liver worms as clonorchis sinensis; risk factors for black pigment gallstone formation are hyperbilirubinbilia, chronic liver insufficiency, chronic hemolysis, pathologic enterohepatic cycling of unconjugated bilirubin, genetic factors, adenomyomatosis of the gallbladder and gallbladder hypokinesis [3, 4]. Cholesterol gallstones formations have been related to metabolic syndrome epidemic and gallbladder hypomotility [4]. Cholesterol gallstones and

A. Cariati

andrea.cariati@libero.it

1 Liver Transplant and Hepatobiliary Surgey Unit, IRCCS-San MARTINO -IST -University Hospital, Monoblocco $4^{\circ}$ Ponente, Largo Rosanna Benzi 10, 16132 Genoa, Italy

2 University of Siena, Siena, Italy

3 Multimedica Hospital, Milan, Italy black pigment gallstones usually form within gallbladder and subsequently migrate in common bile duct except in case of biliary strictures. In conclusion, the main mechanism for brown pigment gallstones formation after gastrectomy is likely to be bactibilia related to the duodenal excluding reconstruction procedures as Billroth II of Roux en Y reconstructions, but the main mechanism for post-gastrectomy cholesterol or black pigment gallstones formation has been gallbladder hypokinesis. Moreover, since almost one-third of post-gastrectomy gallstones is likely to be radiolucent ("pure" cholesterol gallstones), TC abdominal scan follow-up could be inable to detect them and so the incidence of this type of gallstones, at least before becoming secondary calcified, has been underestimated; in addition, these gallstones could benefit from oral dissolution therapy with UDCA or TUDCA if cholecystectomy is not taken in account. In conclusion, in order to prevent the onset of symptomatic gallstones in patients that underwent gastrectomy with duodenal excluding reconstruction procedures, it could be proposed to perform, at the same time, prophylactic cholecystectomy, but further evidence based studies on larger casuistries need to confirm or not benefits from this strategy.

\section{References}

1. Kimura J, Kunisaki C, Takagawa R et al (2016) Is routine prophylactic cholecystectomy necessary during gastrectomy for gastric cancer? World J Surg. doi:10.1007/s00268-016-3831-4

2. Cetta F, Calomino N, Malerba M et al (1993) Every type of gallstones can form after total gastrectomy and total parenteral nutrition. Gastroenterology 104(4 Suppl):A353

3. Vitek L, Carey MC (2012) New pathophysiological concepts underlying pathogenesis of pigment gallstones. Clin Res Hepatol Gastroenterol 31:122-129

4. Cariati A (2015) Gallstone classification in Western countries. Indian J Surg 77(Suppl 2):S376-S380

5. Cetta $F(1991)$ The role of bacteria in pigment gallstone disease. Ann Surg 213(4):315-326 